\title{
A note on the coefficient array of a generalized Fibonacci polynomial
}

\author{
A. G. Shannon \\ Warrane College, the University of New South Wales \\ Kensington, NSW 2033, Australia \\ e-mail: t . shannon@warrane.unsw . edu . au \\ Ömür Deveci \\ Department of Mathematics, Faculty of Science \& Letters, Kafkas University \\ 36100 Kars, Turkey \\ e-mail: odeveci36@hotmail.com
}

Received: 29 April 2020

Accepted: 11 October 2020

Abstract: A particular version of a Fibonacci polynomial is presented and the coefficients are tabulated to bring out some of their number theory properties with known results. Generalizations of the fundamental and primordial Lucas sequences are used in the proofs.

Keywords: Fibonacci numbers and polynomials, Lucas numbers, Bernoulli polynomials, Falling factorial coefficients, Umbral calculus.

2010 Mathematics Subject Classification: 11B39, 11B68.

\section{Introduction}

In a previous paper [9], we defined a generalized Fibonacci polynomial by

$$
u_{n}(x)=\sum_{k=0}^{n} u_{n-k} \frac{n !}{k !} x^{k}
$$

with $P_{0}$ (see below) set as unity for notational convenience, and $\left\{u_{n}\right\}$ and $\left\{v_{n}\right\}$ are generalized arbitrary order $r$ number sequences defined formally by

$$
\begin{gathered}
u_{n}=\sum_{j=1}^{r}(-1)^{j+1} P_{j} u_{n-j}, \quad n>0, \\
u_{n}=1, n=0, \\
u_{n}=0, n<0,
\end{gathered}
$$


and

$$
\begin{aligned}
& v_{n}=\sum_{j=1}^{r}(-1)^{j+1} P_{j} v_{n-j}, \quad n \geq 0, \\
& v_{n}=\sum_{j=1}^{r} \alpha_{j}^{n}, 0 \leq n<r, \\
& v_{n}=0, n<0,
\end{aligned}
$$

where the $P_{j}$ are arbitrary integers and $\alpha_{j}$ are the roots, assumed distinct of the associated auxiliary equation

$$
0=x^{r}-\sum_{j=1}^{r}(-1)^{j+1} P_{j} x^{r-j}
$$

which is associated with the homogeneous arbitrary order linear recurrence relations which the generalized Fibonacci numbers satisfy. For example, when $r=2$, we have

$$
u_{n}=P_{1} u_{n-1}-P_{2} u_{n-2}
$$

or $\left\{u_{n}\right\} \equiv\left\{\left(\left(1, P_{1} ; P_{1}, P_{2}\right)\right\}\right.$ in Horadam's notation [5]. These are the Lucas fundamental numbers [6] and the $\left\{v_{n}\right\} \equiv\left\{\left(\left(2, P_{1} ; P_{1}, P_{2}\right)\right\}\right.$ correspond to the Lucas primordial numbers which can be readily shown to satisfy

$$
v_{n}=\sum_{j=1}^{r} \alpha_{j}^{n}, \text { for all } n \geq 0 .
$$

When $P_{1}=-P_{2}=1$, we get the Fibonacci numbers $\left\{u_{n}\right\} \equiv\left\{F_{n+1}\right\}$ and the ordinary Lucas numbers $\left\{u_{n}\right\} \equiv\left\{L_{n}\right\}$, the principal properties of which can be found in Hoggatt [3]. There are many variations of Fibonacci polynomials in the literature with a common theme that all polynomials can be expressed in terms of appropriately defined Fibonacci polynomials [4, 8] which we demonstrate for the subject of this paper in Section 3 below. Here we propose merely to consider some aspects of the coordinates in (1.1) from some specific examples of (1.1).

\section{Preliminary results}

We can develop another result between Lucas' primordial and fundamental sequences. To do this we establish a recurrence relation for the generalized Fibonacci polynomial (1.1) with the use of a result from [9]:

$$
u_{n+1}(x)=x u_{n}(x)+\sum_{j=0}^{n} n{ }^{j} v_{j+1} u_{n-j}(x)
$$

in which $n \underline{j}$ is the falling factorial coefficient.

Proof: Since from [9] 


$$
\begin{gathered}
\sum_{n=0}^{\infty} u_{n}(x) \frac{t^{n}}{n !}=\exp \left(x t+\sum_{m=1}^{\infty} v_{m} \frac{t^{m}}{m}\right) \\
\frac{\partial}{\partial t} \sum_{n=0}^{\infty} u_{n}(x) \frac{t^{n}}{n !}=\sum_{n=0}^{\infty} u_{n+1}(x) \frac{t^{n}}{n !}
\end{gathered}
$$

and

$$
\begin{array}{r}
\frac{\partial}{\partial t} \exp \left(x t+\sum_{m=1}^{\infty} v_{m} \frac{t^{m}}{m}\right)=\left(\exp \left(x t+\sum_{m m=1}^{\infty} v_{m} \frac{t^{m}}{m}\right)\right)\left(\frac{\partial}{\partial t}\left(x t+\sum_{m=1}^{\infty} v_{m} \frac{t^{m}}{m}\right)\right) \\
=\left(x+\sum_{m=0}^{\infty} v_{m+1} t^{m}\right) \exp \left(x t+\sum_{m=1}^{\infty} v_{m} \frac{t^{m}}{m}\right)
\end{array}
$$

we have that

$$
\begin{aligned}
\sum_{n=0}^{\infty} u_{n+1}(x) \frac{t^{n}}{n !} & =\left(x+\sum_{m=0}^{\infty} v_{m+1} t^{m}\right) \sum_{n=0}^{\infty} u_{n}(x) \frac{t^{n}}{n !} \\
& =\sum_{n=0}^{\infty} x u_{n}(x) \frac{t^{n}}{n !}+\sum_{m=0}^{\infty} v_{m+1} t^{m} \sum_{n=0}^{\infty} u_{n}(x) \frac{t^{n}}{n !} \\
& =\sum_{n=0}^{\infty} x u_{n}(x) \frac{t^{n}}{n !}+\sum_{n=0}^{\infty} \sum_{j=0}^{n} n^{j} v_{j+1} u_{n-j}(x) \frac{t^{n}}{n !}
\end{aligned}
$$

which yields the required result when we equate coefficients. For example, when $x=0$, Equation (2.1) becomes

$$
u_{n+1}=\sum_{j=0}^{\infty} v_{j+1} u_{n-j} /(n+1)
$$

since $n !=n \underline{j}(n-j) !$ and when $r=2$ and $n=1$, the right-hand side of (2.2) reduces to

$$
\begin{aligned}
\frac{1}{2} \sum_{j=0}^{1} v_{j+1} u_{1-j} & =\frac{1}{2}\left(v_{1} u_{1}+v_{2} u_{0}\right) \\
& =\frac{1}{2}\left(P_{1} P_{1}+\left(P_{1}^{2}-2 P_{2}\right)\right) \\
& =P_{1}^{2}-P_{2} .
\end{aligned}
$$

More specifically, for $r=2, P_{1}=-P_{2}=1, v_{n}=L_{n}, u_{n-1}=F_{n}$, the ordinary Lucas and Fibonacci numbers, and Equation (2.1) becomes

$$
n F_{n+1}=4 F_{5}=20
$$

and 


$$
\begin{aligned}
\sum_{j=0}^{3} L_{j+1} F_{n-j} & =L_{1} F_{4}+L_{2} F_{3}+L_{3} F_{2}+L_{4} F_{1} \\
& =1 \times 3+3 \times 2+4 \times 1+7 \times 1 \\
& =20 .
\end{aligned}
$$

Now

$$
\begin{aligned}
\sum_{n=0}^{\infty} u_{n}(x) \frac{t^{n}}{n !} & =(\exp (x t))\left(\exp \left(\sum_{m=1}^{\infty} v_{m} \frac{t^{m}}{m}\right)\right) \\
& =\sum_{k=0}^{\infty} x^{k} \frac{t^{k}}{k !} \sum_{j=0}^{\infty} u_{j} t^{j} \\
& =\sum_{n=0}^{\infty} \sum_{k=0}^{n}\left(\frac{n !}{k !}\right) u_{n-k} x^{k} \frac{t^{n}}{n !}
\end{aligned}
$$

so that on equating coefficients of $t^{n}$ we obtain

$$
u_{n}(x)=\sum_{k=0}^{n} n \frac{n-k}{} u_{n-k} x^{k}
$$

and

$$
u_{n}(x)=\sum_{k=0}^{n}\left(\begin{array}{l}
n \\
k
\end{array}\right) u_{n}(0) x^{k}
$$

Then

$$
u_{0}(x)=u_{0}=1
$$

\section{Generalized polynomials}

Formula (2.4) can be written in the suggestive form

$$
u_{n}(x)=\left(x+u_{n}(0)\right)^{n},
$$

which is analogous to the well-known

$$
B_{n}(x)=\left(x+B_{n}(0)\right)^{n}
$$

for the Bernoulli polynomials, and wherein it is understood that after expansion of the right member, terms of the form $a^{k}$ are replaced by terms of the form $a_{k}$, as in the umbral calculus [7]. We now find that with successive differentiation we can obtain in turn that

$$
\begin{aligned}
D^{2} u_{n}(x) & =D\left(D u_{n}(x)\right) \\
& =n D u_{n-1}(x) \\
& =n(n-1) u_{n-2}(x),
\end{aligned}
$$

and so on, so that

$$
D^{n} u_{n}(x)=n ! u_{0}(x)
$$


and then the not surprising result

$$
D^{n} u_{n}(x)=n ! .
$$

It follows then that any polynomial can be expanded in a series of these generalized polynomials. The proof is as follows:

$$
\begin{aligned}
\exp (x t) & =\left(\exp \left(-\sum_{m=1}^{\infty} v_{m} \frac{t^{m}}{m}\right)\right)\left(\sum_{n=0}^{\infty} u_{n}(x) \frac{t^{n}}{n !}\right) \\
& =\left(1+\sum_{j=1}^{r}(-1)^{j} P_{j} t^{j}\right)\left(\sum_{n=0}^{\infty} u_{n} \frac{t^{n}}{n !}\right) .
\end{aligned}
$$

That is, if $P_{0}=1$ as set earlier, then

$$
\sum_{n=0}^{\infty} x^{n} \frac{t^{n}}{n !}=\sum_{n=0}^{\infty} \sum_{j=0}^{r}(-1)^{j} P_{j} u_{n-j}(x) n-\frac{t^{n}}{n !} .
$$

Equate coefficients of the powers of $t$, and

$$
x^{n}=\sum_{j=0}^{r}(-1)^{j} n^{j} P_{j} u_{n-j}(x) .
$$

It follows from this that the first few generalized Fibonacci polynomial examples of (1.1) are

$$
\begin{aligned}
u_{0}(x) & =1 \\
& =u_{0} \\
u_{1}(x) & =x+P_{1} \\
& =u_{0} x+u_{1} \\
u_{2}(x) & =x^{2}+2 P_{1} x+2\left(P_{1}^{2}-P_{2}\right) \\
& =u_{0} x^{2}+2 u_{1} x+2 u_{2}, \\
u_{3}(x) & =x^{3}+3 P_{1} x^{2}+6\left(P_{1}^{2}-P_{2}\right) x+6\left(P_{1}^{3}-2 P_{1} P_{2}+P_{3}\right) \\
& =u_{0} x^{3}+3 u_{1} x^{2}+6 u_{2} x+6 u_{3},
\end{aligned}
$$

which agree with the first few examples of equations (2.3) and (2.4). The coefficient array is set out in Table 1, from which Tables 2 and 3 are in turn drawn.

\begin{tabular}{|l|c|c|c|c|c|c|c|c|}
\hline & $\mathbf{0}$ & $\mathbf{1}$ & $\mathbf{2}$ & $\mathbf{3}$ & $\mathbf{4}$ & $\mathbf{5}$ & $\mathbf{6}$ & $\mathbf{7}$ \\
\hline $\mathbf{0}$ & 1 & & & & & & & \\
\hline $\mathbf{1}$ & 1 & 1 & & & & & & \\
\hline $\mathbf{2}$ & 1 & 2 & 2 & & & & & \\
\hline $\mathbf{3}$ & 1 & 3 & 6 & 6 & & & & \\
\hline $\mathbf{4}$ & 1 & 4 & 12 & 24 & 24 & & & \\
\hline $\mathbf{5}$ & 1 & 5 & 20 & 60 & 120 & 120 & & \\
\hline $\mathbf{6}$ & 1 & 6 & 30 & 120 & 360 & 720 & 720 & \\
\hline $\mathbf{7}$ & 1 & 7 & 42 & 210 & 840 & 2520 & 5040 & 5040 \\
\hline
\end{tabular}

Table 1. Polynomial coefficients [Sloane [7] A122851] 
The elements of this table can lead to a variety of relationships between the generalized Lucas fundamental and primordial sequences if, in the coefficient array, we make use of

$$
n \frac{n-k}{=} n(n-1)^{(n-1)-k} .
$$

\begin{tabular}{|c|c|c|c|c|c|c|c|c|c|}
\hline Sequences & $\mathbf{0}$ & $\mathbf{1}$ & $\mathbf{2}$ & $\mathbf{3}$ & $\mathbf{4}$ & $\mathbf{5}$ & $\mathbf{6}$ & $\mathbf{7}$ & Sloane [7] \\
\hline Diagonal & 1 & 1 & 2 & 3 & 6 & 11 & 24 & 51 & A122852 [11] \\
\hline Row & 1 & 2 & 5 & 16 & 65 & 326 & 1957 & 13700 & A $000522[2]$ \\
\hline
\end{tabular}

Table 2. Diagonal and row sequences

The references are included here because they can take the interested reader to conjectures related to the topics of this paper.

\begin{tabular}{|c|c|c|c|c|c|c|c|c|c|}
\hline $\begin{array}{l}\downarrow \text { Partial } \\
\text { Column }\end{array}$ & \multicolumn{9}{|c|}{ Sloane } \\
\hline 0 & 1 & 2 & 3 & 4 & 5 & 6 & 7 & 8 & $0 ! \times$ A000027 \\
\hline 1 & 1 & 3 & 6 & 10 & 15 & 21 & 28 & & $1 ! \times$ A230364 \\
\hline 2 & 2 & 8 & 20 & 40 & 70 & 112 & & & $2 ! \times$ A000292 \\
\hline 3 & 6 & 30 & 90 & 210 & 420 & & & & $3 ! \times$ A000332 \\
\hline 4 & 24 & 144 & 504 & 1344 & & & & & $4 ! \times$ A000389 \\
\hline
\end{tabular}

Table 3. Partial column sequences

The relations among the generalized Lucas sequences make these neat relations seem obvious since the first column of this table consists of the factorial numbers (Sloane A000142), but they do take the interested reader further afield [10]. For instance, by inserting alternating positive and negative signs into this series one gets a divergent series which can lead into Borel summation techniques [1].

\section{References}

[1] Borel, É. (1899). Mémoire sur les séries divergentes, Annales scientifiques de l'École Normale Supérieure. Serie 3, 16(1), 9-131.

[2] Hardy, G. H., \& Ramanujan, S. (1917). Asymptotic formulae in combinatory analysis. Proceedings of the London Mathematical Society. Series 2, 16(1), 75-115.

[3] Hoggatt, V. E. Jr. (1969). Fibonacci and Lucas Numbers. Boston, MA: Houghton Mifflin.

[4] Hoggatt, V. E, Jr., \& Bicknell, M. (1973). Roots of Fibonacci polynomials. The Fibonacci Quarterly. 11(3), 271-274.

[5] Horadam, A. F. (1965). Basic properties of a certain generalized sequence of numbers. The Fibonacci Quarterly. 3(3), 161-176. 
[6] Lucas, E. (1878). Théorie des Fonctions Numériques Simplement Périodiques. American Journal of Mathematics. 1, 184-240.

[7] Rota, G.-C., Kahaner, D., \& Oslyzko, A. (1975). Finite Operator Calculus. New York: Academic Press.

[8] Shannon, A. G. (1975). Fibonacci analogs of the classical polynomials. Mathematics Magazine. 48(3), 123-130.

[9] Shannon, A. G., \& Deveci, O. (2018). Some generalized Fibonacci and Hermite polynomials. JP Journal of Algebra, Number Theory and Applications. 40(4), 419-427.

[10] Singh, D. (1952). The numbers $L(m, n)$ and their relations with prepared Bernoulli and Eulerian numbers. Mathematics Student. 20(1), 66-70.

[11] Sloane, N. J. A. (1973). A Handbook of Integer Sequences. New York: Academic Press. 